\title{
The Systematics of Intracluster Starlight
}

\author{
R. Ciardullo \\ Department of Astronomy and Astrophysics, Penn State University, \\ University Park, PA, USA \\ J.C. Mihos, J.J. Feldmeier \\ Department of Astronomy, Case Western Reserve University, \\ Cleveland, $\mathrm{OH}, \mathrm{USA}$
P.R. Durrell, S. Sigurdsson
Department of Astronomy and Astrophysics, Penn State University, University Park, PA, USA

\begin{abstract}
Surveys for intracluster planetary nebulae and red giants have been performed in a variety of cluster environments, from the wellmixed regions of the Virgo and Fornax Clusters, to the low-density areas of the M81 Group. When coupled with the results of Abell Cluster surface photometry and intracluster supernova detections, these surveys provide clues to the systematics of the intracluster light phenomenon. We analyze the results of the intracluster surveys performed to date and show how the fraction of intracluster stars depends on parameters such as cluster richness and rate of galaxy interaction. We also compare our results to numerical predictions and discuss avenues for future research.
\end{abstract}

Recent surveys have yielded some remarkable results about the systematics of intracluster light. While searches for intracluster red giants (IRGB) and planetary nebulae (IPN) in the poor M81 and Leo I groups have found a negligible number of such objects, similar observations in the small clusters of Fornax and Virgo have fixed the percentage of intracluster stars at between $10 \%$ and $20 \%$. The latter number is similar to the fraction of intracluster light found in a dozen rich Abell Clusters via ultra-low surface brightness CCD photometry. Although such photometric measurements carry a considerable uncertainty, the value of $\sim 15 \%$ is also confirmed by a SN Ia survey of 100 rich Abell Clusters between $0.06<z<0.2$. Figure 1 summarizes these results.

One surprising aspect of the figure is the lack of correlation between intracluster stars and Bautz-Morgan type. All clusters are expected to eventually form a cD galaxy, so a cluster's Bautz-Morgan classification should be a measure of its evolutionary state. Yet there is no evidence that centrally condensed systems have more intracluster stars than less organized clusters.

Equally interesting is the distinctive break in the intracluster star fraction at $\sim 10^{11} \mathrm{~L}_{\odot}$. Small groups such as M81 and Leo I have almost no intracluster stars ( $\lesssim 2 \%$ ), while larger systems have $\sim 20 \%$ of their stellar mass in intracluster space. This dichotomy is even more striking when one considers that Leo I 

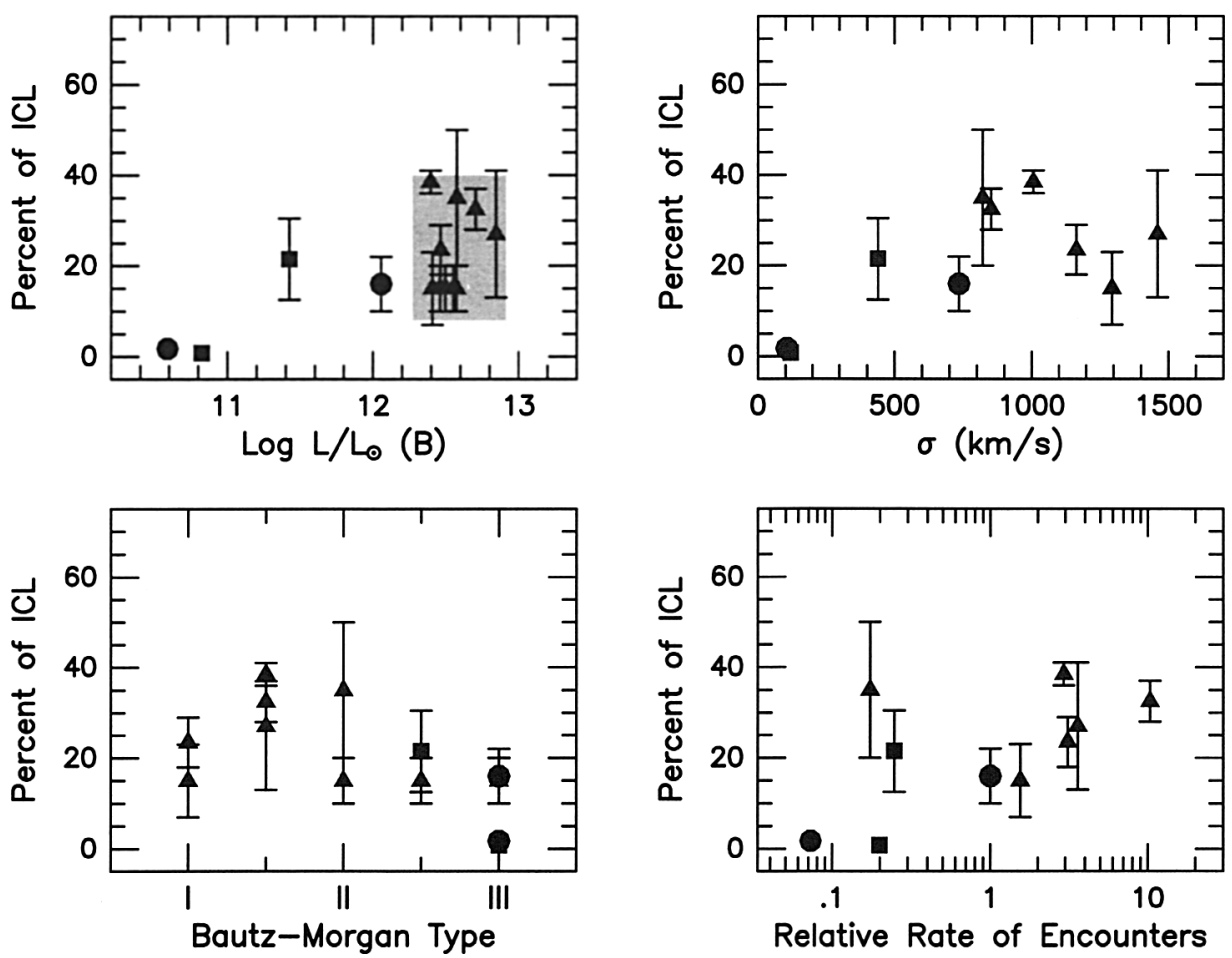

Figure 1. The fraction of intracluster stars as a function of cluster $B$-band luminosity, velocity dispersion, Bautz-Morgan type, and frequency of galaxy encounters. Squares indicate IPN measurements, circles represent IPN and IRGB data, and triangles show the results of CCD surface photometry. The shadowed area illustrates a determination based on a survey for SN Ia.

possesses intracluster $\mathrm{H} \mathrm{I}$ gas and an E/S0 galaxy fraction that is similar to that seen in the larger systems of Fornax and Virgo.

It may be possible to explain the data if most intracluster stars originate in spiral galaxies and if the primary source of heating is the tidal field of the cluster. The former assumption is probably valid, since the mass contained in dwarf spheroidals is negligible, but the latter is problematic, as galaxy-galaxy and galaxy-subgroup encounters should also be important. Nevertheless, the lack of correlation in the fourth panel of the figure supports this idea. If we model spiral galaxies as exponential stellar disks with (massive) isothermal dark matter halos, and assume that in a cluster with velocity dispersion $\sigma_{0}$, the stars outside scale length $r_{t}$ are stripped, then the relative fraction of stripped stars in a similar-size cluster with dispersion $\sigma_{\mathrm{cl}}$ will be proportional to $\exp \left\{-r_{t}\left(\frac{\sigma_{0}}{\sigma_{\mathrm{cl}}}-1\right)\right\}$. This very simple analysis suggests that in the regime where $r_{t}$ is large (i.e., where only the outermost galactic stars are being stripped), the fraction of intracluster light is a sensitive function of cluster velocity dispersion. Surveys of intermediate clusters with $L \sim 10^{11} \mathrm{~L}_{\odot}$ and $\sigma \sim 200 \mathrm{~km} \mathrm{~s}^{-1}$ could test this hypothesis. 